\title{
Involvement of endoplasmic reticulum stress and p53 in IncRNA MEG3-induced human hepatoma HepG2 cell apoptosis
}

\author{
RUI-PEI CHEN ${ }^{1}$, ZHEN-LUN HUANG ${ }^{1}$, LI-XUAN LIU ${ }^{1}$, MENG-QI XIANG ${ }^{1}$, \\ GUO-PING LI ${ }^{1}$, JIA-LIN FENG ${ }^{2}$, BIN LIU $^{3}$ and LING-FEI WU ${ }^{1}$ \\ Departments of ${ }^{1}$ Gastroenterology, ${ }^{2}$ Information and ${ }^{3}$ Neurosurgery, The Second Affiliated Hospital, \\ Shantou University Medical College, Shantou, Guangdong 515041, P.R. China
}

Received April 10, 2016; Accepted May 27, 2016

DOI: $10.3892 / o r .2016 .4919$

\begin{abstract}
Long non-coding RNAs (lncRNAs) play important roles in diverse biological processes. Although downregulation of lncRNA maternally expressed gene 3 (MEG3) has been identified in several types of cancers, little is known concerning its biological role and regulatory mechanism in hepatoma. Our previous studies demonstrated that MEG3 induces apoptosis in a p53-dependent manner. The aim of the present study was to determine whether endoplasmic reticulum (ER) stress is involved in MEG3-induced apoptosis Recombinant lentiviral vectors containing MEG3 (Lv-MEG3) were constructed and transfected into HepG2 cells. A 3-(4,5-dimethylthiazol-2-yl)-2,5-diphenyltetrazolium bromide (MTT) assay, RT-PCR, flow cytometry, western blot analysis, immunofluorescence and immunohistochemistry were applied. Transfected HepG2 cells were also transplanted into nude mice, and the tumor growth curves were determined. The results showed that the recombinant lentivirus of MEG3 was transfected successfully into the HepG2 cells and the expression level of MEG3 was significantly increased. Ectopic expression of MEG3 inhibited HepG2 cell proliferation in vitro and in vivo, and also induced apoptosis. Ectopic expression of MEG3 increased ER stress-related proteins 78-kDa glucose-regulated protein (GRP78), inositol-requiring enzyme 1 (IRE1), RNA-dependent protein kinase-like ER kinase (PERK), activating transcription factor 6 (ATF6), $\mathrm{C} / \mathrm{EBP}$ homologous protein (CHOP), caspase-3, as well as p53 and NF- $\kappa \mathrm{B}$ expression accompanied by NF- $\mathrm{\kappa B}$ translocation from the cytoplasm to the nucleus. Furthermore, inhibition of NF- $\kappa \mathrm{B}$ with Bay11-7082 decreased p53 expression in the MEG3-transfected cells. These results indicate that MEG3
\end{abstract}

Correspondence to: Professor Ling-Fei Wu, Department of Gastroenterology, The Second Affiliated Hospital, Shantou University Medical College, 69 Dongxia Road, Shantou, Guangdong 515041, P.R. China

E-mail: lingfeiwu@21cn.com

Key words: lncRNA MEG3, hepatocelluar carcinoma, endoplasmic reticulum stress, $\mathrm{NF}-\kappa \mathrm{B}$, caspase- 3 , apoptosis, $\mathrm{p} 53$ inhibits cell proliferation and induces apoptosis, partially via the activation of the ER stress and p53 pathway, in which NF- $\kappa \mathrm{B}$ signaling is required for $\mathrm{p} 53$ activation in ER stress.

\section{Introduction}

Recent studies have shown that approximately $80 \%$ of the human genome is transcribed to RNA, with only $2 \%$ being responsible for protein coding. According to their size, non-coding RNAs (ncRNAs) are classified into small and long ncRNAs. Long non-coding RNAs (lncRNAs) are a class of RNA molecules with $>200$ nucleotides that function as RNAs with little or no protein-coding capacity (1). IncRNAs have been implicated to play a functional role in carcinogenesis and cancer growth (2). There is increasing evidence that lncRNAs participate in a diversity of biological processes. Expression of many lncRNAs is altered and is likely to function in tumorigenesis. The functional diversity and mechanistic role of IncRNAs are currently a field of intense investigation (3).

Maternally expressed gene 3 (MEG3), an IncRNA, is an imprinted gene located on chromosome 14q32.3 that functions as a tumor suppressor $(4,5)$. MEG3 is expressed in many normal human tissues, with the highest expression in brain and pituitary gland. However, its expression is lost or decreased in common human tumors, such as glioma, colon cancer, hepatocellular cancer, non-small cell lung cancer (NSCLC) and meningioma (2,6-9). We and other investigators have demonstrated that promoter hypermethylation or hypermethylation of the intergenic differentially methylated region contributes to the loss of MEG3 expression in tumors (10).

The endoplasmic reticulum (ER) is the intracellular organelle where protein synthesis, folding, and modifications occur, as well as regulation of intracellular $\mathrm{Ca}^{2+}$ homeostasis (11). Various stimuli can disturb ER homeostasis resulting in the accumulation of unfolded or misfolded proteins leading to pathological consequences, generally known as ER stress (12).

Slight stimulation activates an adaptive signaling cascade to counteract the ER stress-associated damage, known as the unfolded protein response (UPR) (13). During UPR, three major stress sensors, comprised of the RNA-dependent protein kinase-like ER kinase (PERK), activating transcription factor 6 (ATF6), and inositol-requiring enzyme 1 (IRE1), reside on the ER membrane and are activated by dissociating 
from the 78-kDa glucose-regulated protein (GRP78). UPR can enhance cell survival (14). However, when the response is not sufficient and ER stress persists, the UPR leads to apoptosis through activation of $\mathrm{C} / \mathrm{EBP}$ homologous protein (CHOP) (15), caspase-12 (16) and/or c-Jun NH2-terminal kinase (JNK) (17).

Our previous study indicated that adenosine induces ER stress and p53 activation, as well as the expression of MEG3 by altering the methylation status of the MEG3 promoter (12), and MEG3 also induces cell apoptosis through the p53-dependent pathway $(10,18)$. Moreover, p53 activation is thought to be related to ER stress (19). However, the biological role of MEG3 has not yet been completely understood; In particular, little is known concerning the regulatory relationship between MEG3 and ER stress. Here, we explored a functional mechanism of MEG3 regulation via activating ER stress and p53 pathway to induce cell apoptosis, and further defined its tumor-suppressor function.

\section{Materials and methods}

Reagents and antibodies. Dulbecco's modified Eagle's medium (DMEM) was purchased from Invitrogen (Carlsbad, CA, USA). Primary antibodies against GRP78, IRE1, PERK, ATF6, p53, and NF-кB were obtained from Santa Cruz Biotechnology, Inc. (Santa Cruz, CA, USA). Primary antibodies against GAPDH and caspase-3 were purchased from Cell Signaling Technology (CST; USA) and CHOP from MengZhouShi Ruiying Biological Technology Co., Ltd. (China). Anti-rabbit and anti-mouse secondary antibodies were purchased from LI-COR Biosciences (USA). Alexa Fluor 555-labeled donkey anti-rabbit IgG was obtained from Abcam (USA). The Annexin V/FITC Apoptosis Detection kit was obtained from Beijing 4A Biotech Co., Ltd. (China). The primer sequences for the MEG3 gene, used to verify transfection efficiency were: forward, CTC AGG CAG GAT CTG GCA TA; and reverse, CCT GGA GTG CTG TTG GAG AA and purchased from Invitrogen. Other materials, such as Tris, SDS and glycine were obtained from Sangon (Shanghai, China).

Cell culture and experimental groups. Human hepatoma HepG2 cells were obtained from the American Type Culture Collection (Manassas, VA, USA) and cultured in DMEM supplemented with $10 \%(\mathrm{v} / \mathrm{v})$ fetal bovine serum (FBS), penicillin $(100 \mathrm{U} / \mathrm{ml})$, and streptomycin $(100 \mathrm{mg} / \mathrm{ml})$ under a humidified atmosphere with $5 \% \mathrm{CO}_{2}$ at $37^{\circ} \mathrm{C}$. The cell culture medium was changed every other day, and cells were passaged at $80-90 \%$ confluency. For the immunofluorescence and immunohistochemical assay, the cells were cultured on coverslips in DMEM for $24 \mathrm{~h}$ before transfection.

For the recombinant lentiviral transfection experiments, HepG2 cells were transfected with a lentiviral vector containing MEG3 (Lv-MEG3) sequence diluted in DMEM with 2\% FBS and $8 \mu \mathrm{g} / \mathrm{ml}$ Polybrene at a multiplicity of infection (MOI) of 100. The cell lines transfected with the empty lentiviral vector (Lenti6.3-MIG) at MOI of 100 served as the control.

Construction of the recombinant lentiviral vectors and HepG2 cell lines stably expressing MEG3. The MEG3 gene was designed and synthesized by means of PCR according to the GenBank of human MEG3 (NR_002766) gene sequence (10).
The purified MEG3 gene fragment was inserted into a lentiviral vector (pLenti6.3-MCS-IRES2-EGFP), and the insertion fragment was identified by PCR, restriction endonuclease analysis and DNA sequencing. The Lv-MEG3 sequence was then transferred into HEK 293FT cells to be packaged into mature lentivirus as Lenti-hMEG3-IRES-EGFP (Lv-MEG3, MEG3).

To obtain cell lines stably expressing MEG3, the lentiviral vector (Lv-MEG3, MEG3) was transfected into HepG2 cells. RT-PCR was performed to examine the mRNA expression level of MEG3.

RNA extraction and RT-PCR assay. HepG2 cells were transfected with Lv-MEG3 or Lenti6.3-MIG for $48 \mathrm{~h}$. Cells were harvested and total RNA was extracted using TRIzol reagent according to the manufacturer's protocol. For reverse transcriptase analysis, $1 \mu \mathrm{g}$ of total RNA was reverse transcribed using an EasyScript First-Strand cDNA Synthesis SuperMix kit. Amplification of MEG3 and GAPDH was performed using $1 \mu \mathrm{l}$ cDNA as a template, $12.5 \mu \mathrm{l} 2 \mathrm{X}$ Taq PCR MasterMix (Tiangen

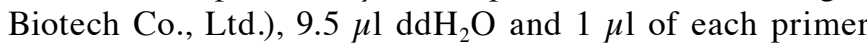
(10 $\mu \mathrm{M}$ stock concentration) in a total volume of $25 \mu \mathrm{l}$. The PCR reaction was performed using the following primers: MEG3 forward, 5'-CTCAGGCAGGATCTGGCATA-3' and reverse, 5'-CCTGGAGTGCTGTTGGAGAA-3'; GAPDH forward, 5'-CACCATCTTCCAGGAGCGA-3' and reverse, 5'-TCAGCAGAGGGGGCAGAGA-3'.

Samples were incubated at $94^{\circ} \mathrm{C}$ for 3 min followed by 38 cycles of denaturation at $94^{\circ} \mathrm{C}$ for $30 \mathrm{sec}$, annealing at $57^{\circ} \mathrm{C}$ for $30 \mathrm{sec}$ and extension at $72^{\circ} \mathrm{C}$ for $1 \mathrm{~min}$, and a final extension step at $72^{\circ} \mathrm{C}$ for $5 \mathrm{~min}$. The PCR products were separated on a $4 \%$ agarose gel and visualized by ethidium bromide staining.

Determination of cell proliferation by MTT assay. Viability of the cell post-transfection was determined using the 3-(4,5-dimethylthiazol-2-yl)-2,5-diphenyltetrazolium bromide (MTT) cell proliferation kit. Cells were inoculated into 96-well plates at $5 \times 10^{3}$ cells/well and allowed to attach for $24 \mathrm{~h}$. The HepG2 cells were transfected with Lv-MEG3 or Lenti6.3-MIG for $48 \mathrm{~h}$. The culture medium was then aspirated and the cells were washed with PBS, followed by incubation with $0.5 \mathrm{mg} / \mathrm{ml}$ of MTT at $37^{\circ} \mathrm{C}$ for $4 \mathrm{~h}$. After incubation, the supernatant was removed and the cells were treated with DMSO to dissolve the formazan reaction product. The concentration of formazan was determined by measuring the absorbance at $540 \mathrm{~nm}$ using an enzyme-linked immunosorbent assay reader (FilterMax F5; Molecular Devices, USA).

Tumor formation assay in a nude mouse model. Five-week-old female athymic BALB/c mice were purchased from Vital River (Beijing, China) and maintained under specific pathogen-free conditions and manipulated according to protocols approved by the Shanghai Medical Experimental Animal Care Commission. HepG2 cells transfected with Lv-MEG3 or Lenti6.3-MIG were harvested, washed with cold PBS and resuspended at a concentration of $3 \times 10^{7}$ cells $/ \mathrm{ml}$. A volume of $0.15 \mathrm{ml}$ each of the suspending cells was subcutaneously injected into either side of the forelimb flank of each nude mouse. Tumor growth was examined every 3 days in mice from the MEG3 $(n=6)$ or control group $(n=6)$, and tumor volumes were calculated using the equation: $\mathrm{V}=0.5 \times \mathrm{D} \times \mathrm{d}^{2}$ 
( $\mathrm{V}$, volume; $\mathrm{D}$, longitudinal diameter; $\mathrm{d}$, latitudinal diameter). Twenty-two days after injection, the mice were euthanized and tumor weights were measured. RT-PCR analysis was performed to detect MEG3 levels in the tumor tissues.

This study was carried out in strict accordance with the recommendations in the Guide for the Care and Use of Laboratory Animals of the National Institutes of Health (20). The protocol was approved by the Committee on the Ethics of Animal Experiments of Shantou University Medical College. All surgery was performed under sodium pentobarbital anesthesia, and all efforts were made to minimize suffering in mice.

Flow cytometric detection ofapoptosis. HepG2 cells transfected with Lv-MEG3 or Lenti6.3-MIG were harvested by trypsinization at $48 \mathrm{~h}$, washed twice with cold PBS, and resuspended in $1 \mathrm{X}$ binding buffer to a concentration of $1 \times 10^{6}$ cells $/ \mathrm{ml}$. The cells were then stained with $5 \mu \mathrm{l}$ Annexin V/FITC and $10 \mu \mathrm{l}$ propidium iodide $(20 \mu \mathrm{g} / \mathrm{ml})$ for $15 \mathrm{~min}$ at room temperature in the dark. Analyses were performed with a BD Accuri ${ }^{\text {TM }}$ C6 Flow Cytometer (BD Biosciences, USA) with the FL1 and FL3 detector.

Western blot analysis. HepG2 cells were harvested at $48 \mathrm{~h}$ after MEG3 transfection, and washed with cold PBS three times. Total cellular protein lysates were prepared with RIPA buffer containing proteinase inhibitors. Nuclear and cytoplasmic protein fractions were prepared using a Nuclear and Cytoplasmic Protein Extraction kit (Beyotime Institute of Biotechnology). Protein concentration was measured using a BCA Protein Assay kit (Thermo Fisher Scientific, USA). Protein $(50 \mu \mathrm{g})$ was separated on $10 \%$ sodium dodecyl sulfate-polyacrylamide gel electrophoresis (SDS-PAGE), and transferred to nitrocellulose membranes. The membranes were blocked at room temperature for $60 \mathrm{~min}$ with $5 \%$ non-fat milk in Tris-buffered saline (pH 7.6; TBS), and incubated with primary antibodies against GAPDH $(1: 1,000)$, GRP78 (1:500), PERK (1:500), IRE1 (1:500), ATF6 (1:500), CHOP (1:1,000), caspase-3 $(1: 1,000)$, p53 $(1: 3,000)$ and NF- $\mathrm{BB}(1: 500)$ in TBS at $4^{\circ} \mathrm{C}$ overnight, followed by TBST (with Tween-20) washes. Membranes were incubated with fluorescent secondary antibodies (LI-COR) coupled to the primary antibody at room temperature in the dark for $1 \mathrm{~h}$, followed by TBST washes, dried with neutral absorbent paper and scanned by Odyssey detection system (LI-COR). Protein expression was analyzed using Quantity One software (Bio-Rad, USA) and normalized to GAPDH (for total cell fraction) or TBP (for nuclear fraction).

Immunofluorescence staining. HepG2 cells were seeded at $4 \times 10^{5}$ cells/well in 6 -well flat bottomed plates and incubated in $10 \%$ FBS-supplemented DMEM for $24 \mathrm{~h}$. Cells were transfected and divided into two groups: the MEG3-transfected group and the control group. Cells were fixed in $4 \%$ paraformaldehyde for 15 min and permeabilized in $0.3 \%$ Triton X-100 for $10 \mathrm{~min}$ at room temperature, and then blocked with $3 \%$ BSA for $30 \mathrm{~min}$. Subsequently, the cells were incubated with the primary antibody against $\mathrm{NF}-\kappa \mathrm{B}$ (anti-p65, 1:100) or with PBS (blank group) at $4^{\circ} \mathrm{C}$ overnight. On the following day, HepG 2 cells were incubated with the Alexa Fluor 555-labeled donkey anti-rabbit IgG (1:100) for $1 \mathrm{~h}$ at $37^{\circ} \mathrm{C}$. The cell nuclei
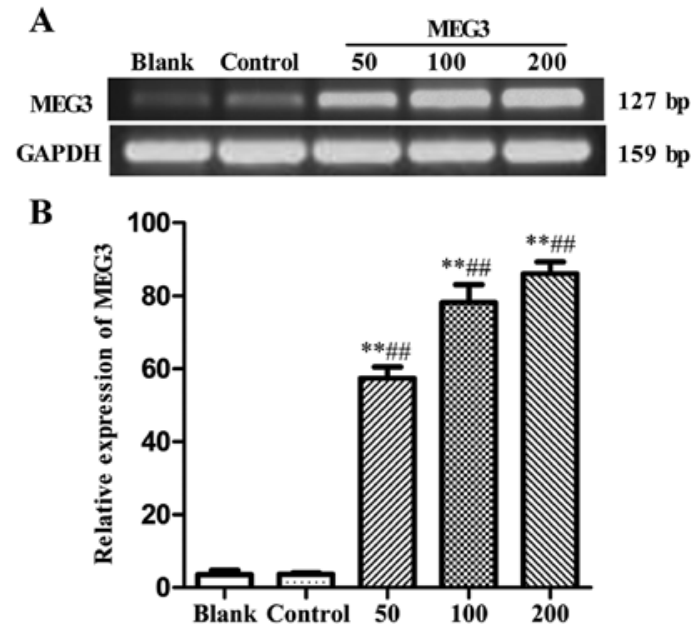

Figure 1. Lv-MEG3 was successfully transfected and a high level mRNA expression of MEG3 was obtained in the HepG2 cells. (A) The mRNA expression levels of MEG3 in the HepG2 cells after MEG3 transfection with the lentivirus containing MEG3 at different MOIs (50, 100, 200), the blank control or the empty lentivirus (Lenti6.3-MIG). (B) Quantitative analysis of the band intensity shown in (A). The bars represent the means \pm SD of five groups; $(\mathrm{n}=3) ;{ }^{*} \mathrm{p}<0.05,{ }^{* *} \mathrm{p}<0.01$ vs. blank group; ${ }^{\#} \mathrm{p}<0.05,{ }^{\# \#} \mathrm{p}<0.01$ vs. control group. Lv-MEG3, lentiviral vector containing MEG3; MEG3, maternally expressed gene 3; MOI, multiplicity of infection.

were stained with DAPI for $10 \mathrm{~min}$. Cells were viewed and captured with a fluorescence microscope (Olympus BX51).

Immunohistochemical staining. For immunochemical analysis, the cells were fixed in $4 \%$ paraformaldehyde for $10 \mathrm{~min}$, permeabilized in $0.3 \%$ Triton X-100 for $10 \mathrm{~min}$ at room temperature, respectively, and then blocked in 3\% BSA for $30 \mathrm{~min}$. Subsequently, the cells were incubated with the primary antibody against NF- $\kappa$ B (anti-p65, 1:50) or with PBS (negative control) at $4^{\circ} \mathrm{C}$ overnight. On the second day, the cells were then incubated with biotinylated anti-mouse (1:400; Jackson ImmunoResearch) at room temperature for $1 \mathrm{~h}$, and then with an avidin-horseradish peroxidase complex (Vectastain ABC kit; Promega, Madison, WI, USA) at room temperature for $30 \mathrm{~min}$, and visualized with amine nickel sulfate-enhanced 3,3'-diaminobenzidine (DAB). Hematoxylin was used to stain the cell nuclei. Sections were observed under a light microscope, and positive staining was shown by the development of brown particles.

Statistical analysis. All experiments were independently performed at least three times. The values are presented as the means \pm SD. Differences were assessed by two-tailed Student's t-test. $\mathrm{P}<0.05$ was considered to indicate a statistically significant result.

\section{Results}

MEG3 expression is increased after Lv-MEG3 transfection in the HepG2 cells. In the present study, we described the generation of a recombinant Lv-MEG3. The pcDNA3.1-MEG3 plasmid vectors and the recombinant lentiviral vectors (Lv-MEG3) were identified using sequencing technique. The particular sequence of MEG3 insertion into vectors was the same as designed. The mRNA expression of MEG3 was observed by RT-PCR. As shown in Fig. 1, compared with 


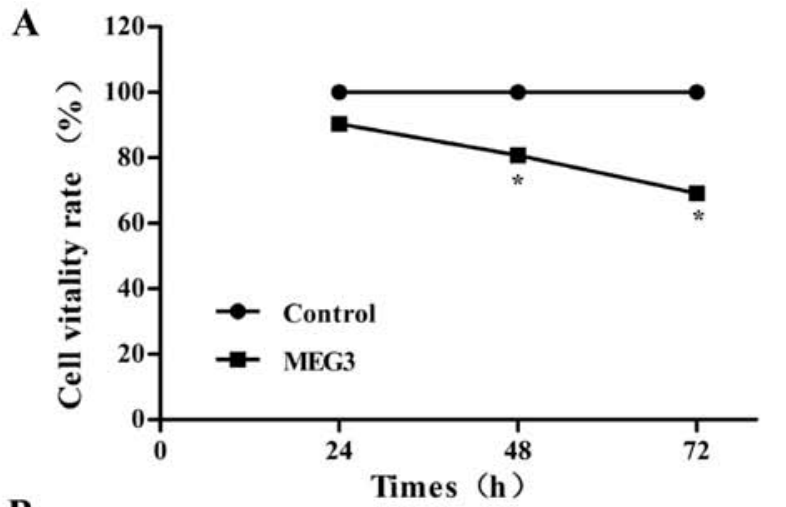

B

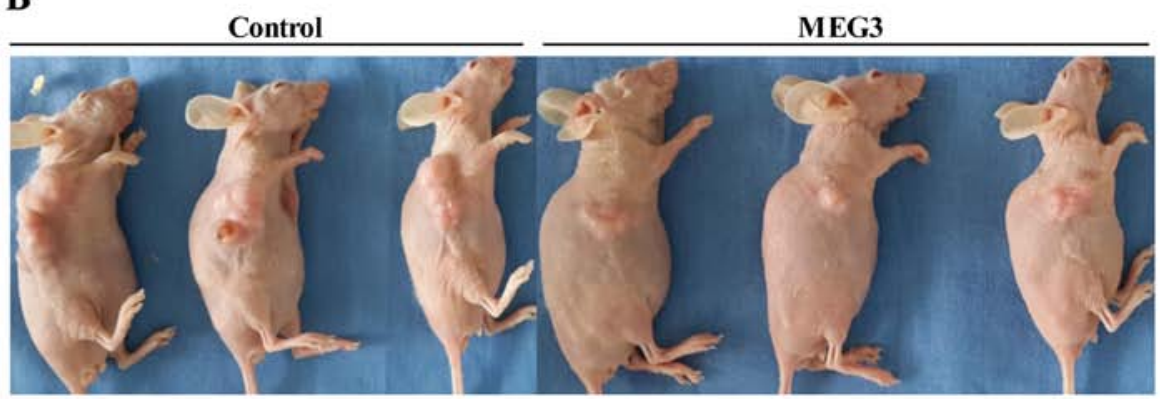

C

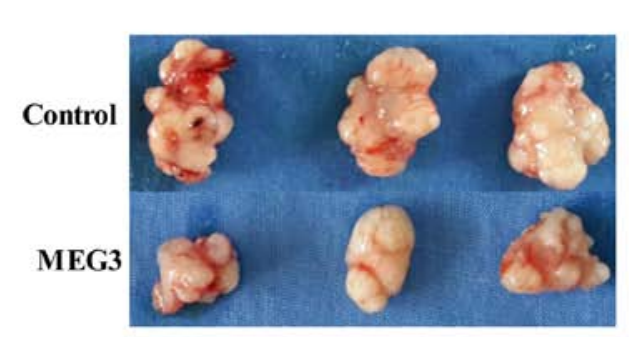

$\begin{array}{ll}1600 \\ \rightarrow & \rightarrow \text { Control } \\ \rightarrow \text { MEG3 }\end{array}$
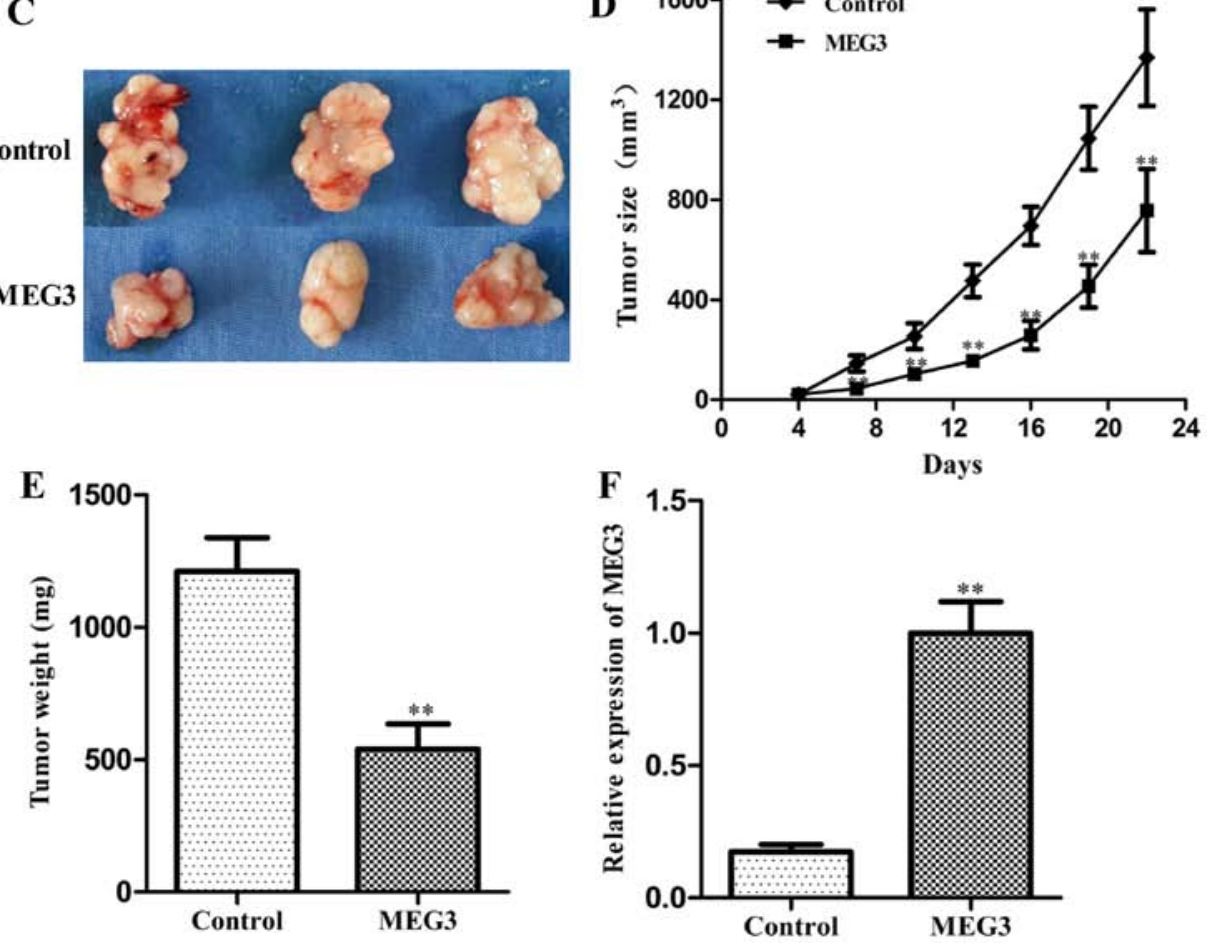

Figure 2. Effects of ectopic expression of MEG3 on tumor growth in vitro and in vivo. (A) MTT assay was performed to determine the proliferation of HepG2 cells. Data represent the means \pm SD from three independent experiments. (B) Effects of ectopic expression of MEG3 on tumor growth in vivo. (C) Tumor sizes were calculated on day 22 after injection of HepG2 cells stably transfected with Lv-MEG3 or the empty vector. (D) Tumor growth curves. Tumor volumes were calculated every 3 days after injection. (E) Tumor weights are represented as the means \pm SD. (F) RT-PCR assay was performed to detect the expression level of MEG3 in the tumor tissues. Data are presented as the means \pm SD; $(n=3) ;{ }^{*} p<0.05,{ }^{* *}$ p $<0.01$ vs. control. MEG3, maternally expressed gene 3; Lv-MEG3, lentiviral vector containing MEG3.

the blank group or empty control group (Lenti6.3-MIG), the mRNA expression of MEG3 was significantly increased after Lv-MEG3 transfection at 50,100 and 200 MOI $(57.44 \pm 3.08,78.13 \pm 4.91$, and $86.09 \pm 3.17 \%$, respectively, vs. $2.32 \pm 0.14$, and $3.58 \pm 1.19 \%$, both $\mathrm{p}<0.01$ ), showing a marked dose-dependent increase in MEG3 expression. This demonstrated that Lv-MEG3 was successfully transfected and a high level of mRNA expression of MEG3 was achieved in the HepG2 cells.

Ectopic expression of MEG3 inhibits hepatoma cancer cell proliferation in vitro and in vivo. To determine whether MEG3 affects the proliferation of HepG2 cells, we performed an MTT assay following transfection of either Lv-MEG3 or 

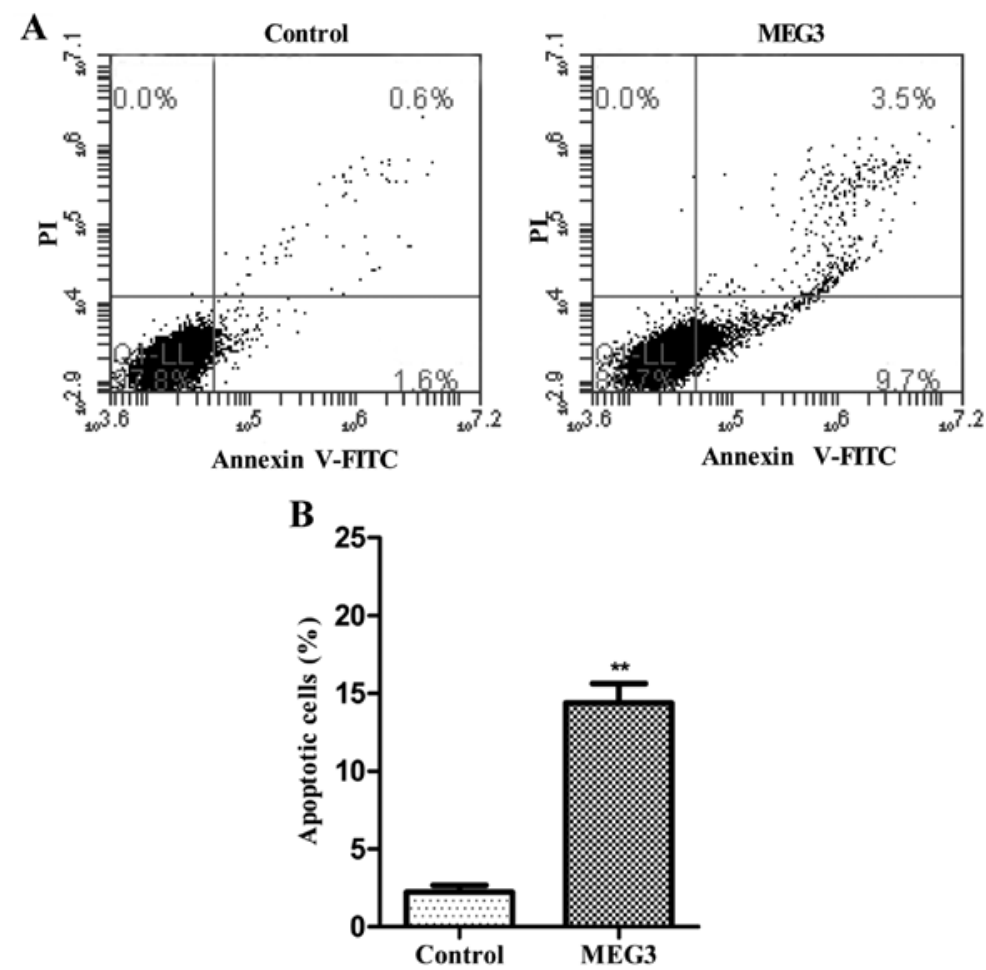

Figure 3. Apoptosis induction by ectopic expression of MEG3 in the HepG2 cell lines. HepG2 cells were transfected with Lv-MEG3 or Lenti6.3-MIG. (A) Apoptosis was detected by flow cytometry based on propidium iodide (y-axis) and Annexin V staining (x-axis). Percentages of apoptotic cells (lower and upper right quadrants) are indicated. (B) Percent of apoptotic cells at $48 \mathrm{~h}$ after transfection. The graph represents the mean percentage of apoptosis $\pm \mathrm{SD}$; $(\mathrm{n}=3) ;{ }^{* *} \mathrm{p}<0.01$ vs. control. MEG3, maternally expressed gene 3; Lv-MEG3, lentiviral vector containing MEG3.

Lenti6.3-MIG. Ectopic expression of MEG3 significantly decreased cell growth by 19.3 and $30.8 \%$ after 48 and $72 \mathrm{~h}$, respectively, compared with the control group (Fig. 2A, $\mathrm{p}<0.05)$. Ectopic expression of MEG3 inhibited cell proliferation in a time-dependent manner. These results suggest that overexpression of MEG3 inhibited HepG2 cell proliferation in vitro.

To explore whether MEG3 inhibits hepatoma growth in vivo, the HepG2 cells transfected with Lv-MEG3 or Lenti6.3-MIG were inoculated into nude mice respectively, and all mice developed xenograft tumors at the injection site. Tumor growth in the Lv-MEG3 group was significantly slower than that in the control group on day 22 after injection (Fig. 2B-D). The average tumor weight in the Lv-MEG3 transfection group was lower than that in control group (Fig. 2E). RT-PCR analysis showed that the mRNA expression level of MEG3 in the Lv-MEG3 transfection group was significantly higher than that in the control group (Fig. 2F). Taken together, these results demonstrated that ectopic expression of MEG3 inhibited hepatoma growth in nude mice.

Ectopic expression of MEG3 induces HepG2 cell apoptosis. To determine whether apoptosis was a contributing factor to cell growth arrest in the MEG3-transfected HepG2 cells, we performed flow cytometric analysis after transfection with Lv-MEG3 or Lenti6.3-MIG. Transfection with Lv-MEG3 increased the fraction of apoptotic cells by $\sim 14.7 \%$ in comparison with cells transfected with the empty lentivirus (Lenti6.3-MIG, Fig. 3). This indicated that overexpression of MEG3 induced HepG2 cell apoptosis in vitro.
Ectopic expression of MEG3 activates the ER stress pathway. To verify whether the MEG3-induced cell apoptosis is related to ER stress, ER stress-relative proteins were detected by western blot analysis. Fig. 4 shows that overexpression of MEG3 not only increased the expression of GRP78, three key proteins of UPR (IRE1, PERK, ATF6), but also increased CHOP, caspase- 3 and 553 expression. The results showed that ectopic expression of MEG3 induced HepG2 cell apoptosis through the ER stress pathway.

Ectopic expression of MEG3 activates $N F-\kappa B$, p53 and causes nuclear translocation of $N F-\kappa B$ protein. In order to further explore the possible relationship between $\mathrm{NF}-\kappa \mathrm{B}$ and $\mathrm{p} 53$, we investigated the distribution of $\mathrm{NF}-\kappa \mathrm{B}$ in the HepG2 cells transfected with MEG3 by immunofluorescence and immunohistochemistry assay. As indicated in Fig. 5A and B, a weak $\mathrm{NF}-\kappa \mathrm{B}$ signal was detected in the control cells and NF- $\kappa \mathrm{B}$ was obviously translocated from the cytoplasm to the nucleus in the MEG3-transfected cells. In order to further validate the results using microscopy, total cell lysates collected from the transfected HepG 2 cells were fractionated to separate the cytoplasmic and nuclear components, and western blot analysis was performed to measure the $\mathrm{NF}-\kappa \mathrm{B}$ protein level only in the nuclear components. A sharp accumulation of $\mathrm{NF}-\kappa \mathrm{B}$ in the nuclear fraction was detected in the cells transfected with MEG3 (Fig. 5C). Moreover, inhibition of NF- $\kappa \mathrm{B}$ by Bay11-7082 decreased the protein expression of both NF- $\kappa \mathrm{B}$ and p53 (Fig. 5D). These results demonstrated that ER stress activates the p53 pathway and p53 expression is mediated by $\mathrm{NF}-\kappa \mathrm{B}$ in MEG3-induced apoptosis. 
A

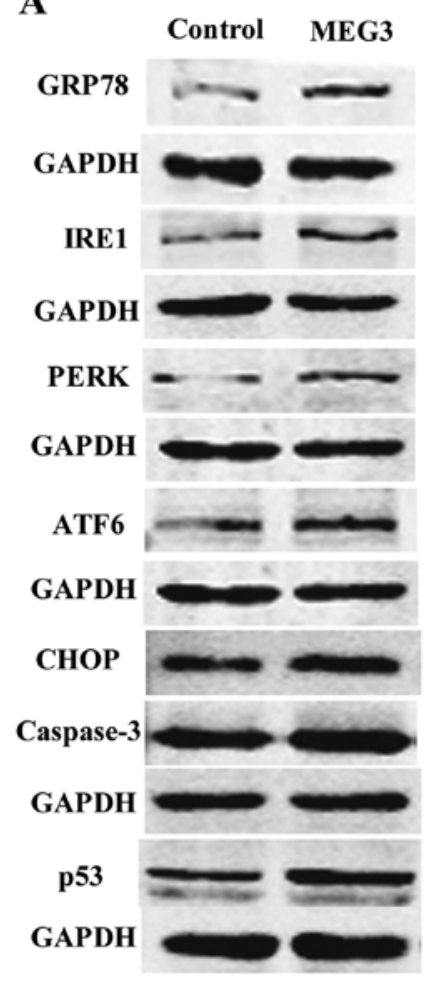

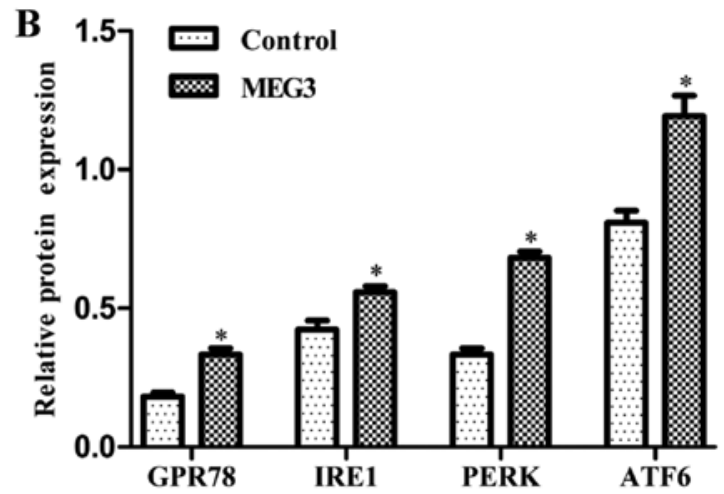

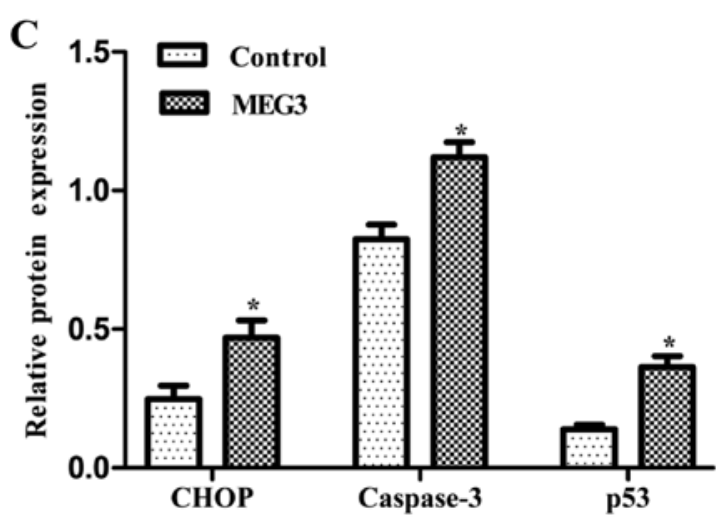

Figure 4. Effects of ectopic MEG3 on protein expression of ER stress-related genes (GRP78, IRE1, PERK, ATF6, CHOP, caspase-3 and p53). (A) Representative images of western blot analysis. The total cellular proteins derived from MEG3-transfected or Lenti6.3-MIG-transfected HepG2 cells were immunoblotted with a panel of antibodies specific for GRP78, IRE1, PERK, ATF6, CHOP, caspase-3, p53 and GAPDH. (B and C) The expression of each index was normalized to the expression level of GAPDH, and the relative change was expressed as a ratio or fold. Data represent the means \pm SD of three independent experiments. ${ }^{*} \mathrm{P}<0.05$ vs. control. MEG3, maternally expressed gene 3; ER, endoplasmic reticulum; GRP78, 78-kDa glucose-regulated protein; IRE1, inositol-requiring enzyme 1; PERK, RNA-dependent protein kinase-like ER kinase; ATF6, activating transcription factor 6; CHOP, C/EBP homologous protein.

\section{Discussion}

Accumulating evidence indicates that MEG3 plays an important role in the formation and progression of HCC. In order to further identify the biological function of MEG3, recombinant lentivirus of MEG3 was constructed and transfected into hepatoma HepG2 cells. The results showed that ectopic expression of MEG3 inhibited HepG2 cell proliferation in vitro (Fig. 2A). Moreover, overexpression of MEG3 decreased tumor growth in nude mice (Fig. 2B-D). FCM analysis further confirmed that the ectopic expression of MEG3 increased apoptosis in the HepG2 cells (Fig. 3), which shows that MEG3 functions as a tumor suppressor.

In the present study, we observed that overexpression of MEG3 significantly increased the relative protein expression of the ER stress pathway. Many studies have indicated that activation of IRE1, ATF6, and PERK represents the standard UPR pathways. Once PERK, ATF6 and/or IRE1 are activated, they initiate an early adaptive response to unfolded proteins that, if the stress is short-lived, can facilitate clearance of the unfolded proteins and cell survival; specifically, inhibition of protein translation, transcriptional induction and increased expression of GRP78 (21). However, with prolonged stress, additional responses are initiated, including caspase-12/caspase-9/caspase-3, ERK/ATF-4/CHOP, IRE1/Ask1/JNK, PERK/eIF2a/NF- $\mathrm{KB}$ and $\mathrm{p} 53$ pathways and these pathways can promote cell apoptosis (22). CHOP is a transcription factor and plays a critical role in ER stress-mediated apoptosis (22). Caspase-3 is a main executioner caspase. In the present study, three key proteins of UPR (IRE1, ATF6, PERK) and chaperone GRP78 were obviously increased in the transfected HepG 2 cells. Furthermore, CHOP, NF-кB, caspase-3 and p53 were also upregulated (Fig. 4). These experimental results demonstrated that MEG3 triggered the ER stress pathway in the HepG2 cells.

Our previous study revealed that MEG3 functions as a tumor-suppressor gene by regulating p53 activation (10). $\mathrm{NF}-\kappa \mathrm{B}$ family members also play a role in regulating the $\mathrm{p} 53$ gene in certain types of stress $(23,24)$. However, the regulatory mechanism of p53 activation in ER stress is still unclear. Studies indicate that persistent ER stress can trigger a switch in the UPR signaling pathways from pro-survival to pro-apoptotic pathways by the PERK/eIF2a/NF- $\mathrm{KB}$ pathways (25). Activation of NF- $\kappa B$ regulates cell death-associated gene expression (26-28). In order to probe the effect of NF- $\kappa B$ on $p 53$ activation, the distribution and expression of NF- $\mathrm{KB}$ protein in the HepG2 cells were detected. In normal states, NF- $\kappa B$ is sequestered in the cytoplasm in an inactive form bound to one of many inhibitory molecules (IкBs). Phosphorylated and ubiquitinated I $\mathrm{KB}$ is degraded by the $26 \mathrm{~S}$ proteasome, leading to the translocation of active NF- $\kappa B$ to the nucleus where it binds to $\kappa \mathrm{B}$ elements and regulates transcription of genes mediating inflammation, carcinogenesis, and pro-apoptotic or anti-apoptotic functions. In this study, the results of the immu- 
A
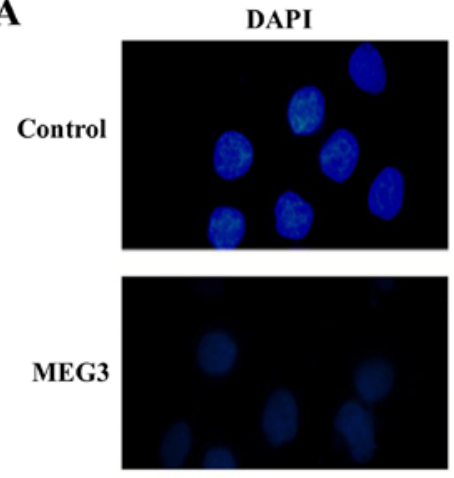

B

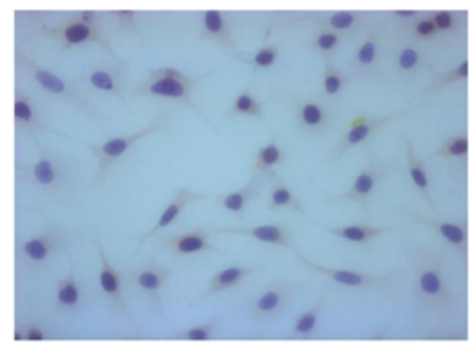

Control
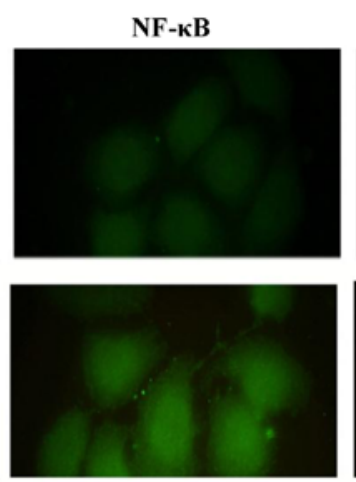
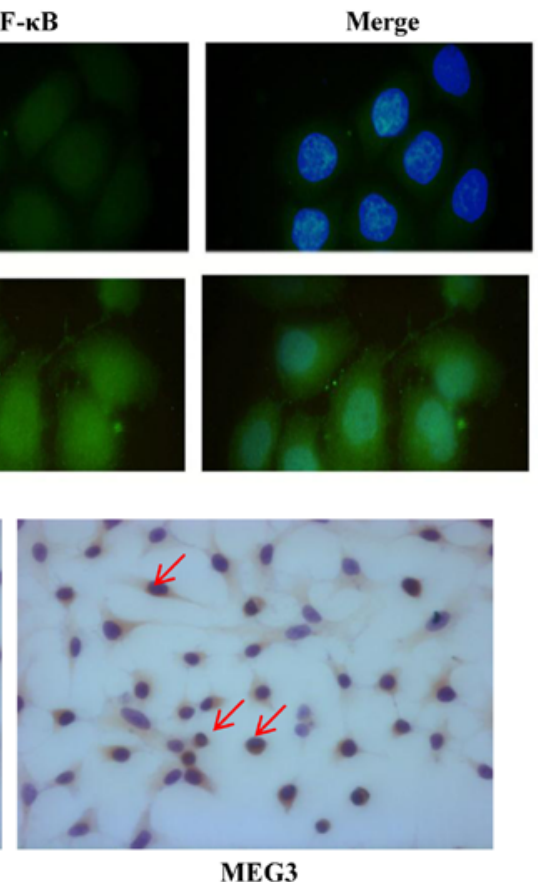

C

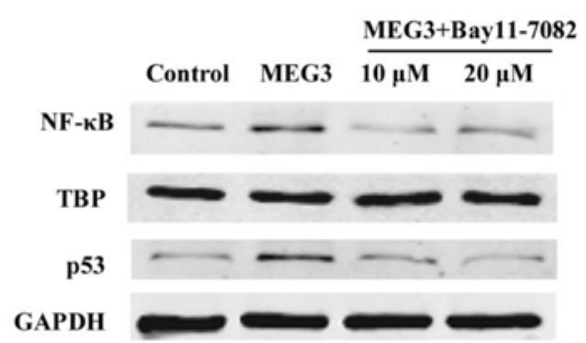

D
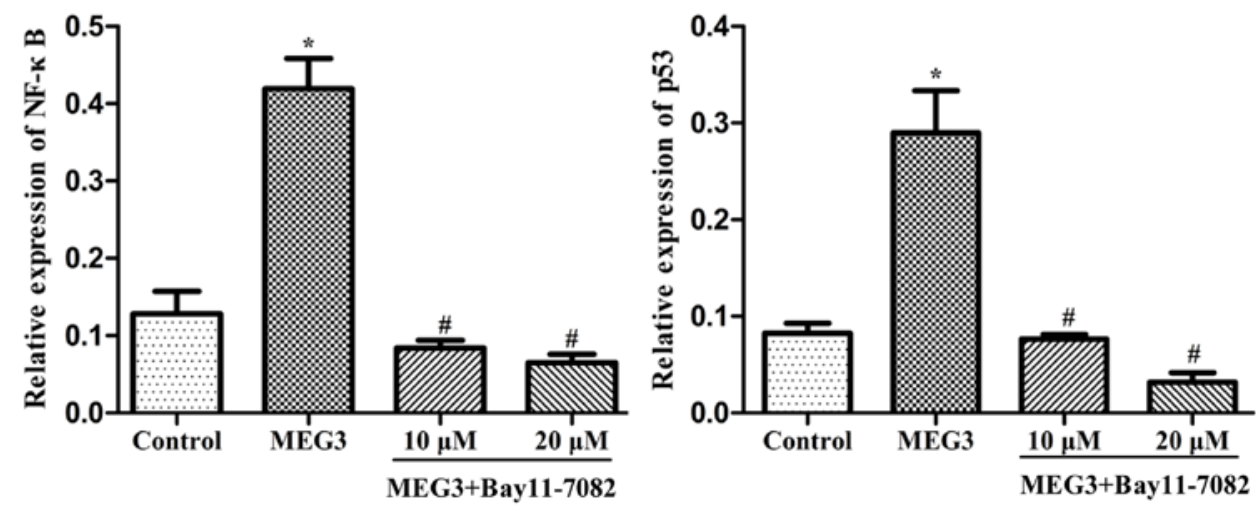

Figure 5. Effects of ectopic MEG3 on the protein expression of NF- $\kappa \mathrm{B}$ and p53 and the role of NF- $\mathrm{B}$ inhibitor on p53 expression. (A) HepG2 cells were transfected with either Lv-MEG3 or Lenti6.3-MIG. The localization of NF- $\mathrm{B}$ was determined by immunofluorescence staining. Cell nuclei were stained with DAPI (blue). (B) The localization of NF- $\mathrm{B}$ was determined by immunocytochemical staining. The red arrows show increasing expression of NF- $\kappa \mathrm{B}$ in the nucleus. (C) After transfection with Lv-MEG3, the cells were then treated with 10 or $20 \mu \mathrm{M}$ Bay11-7082 for $24 \mathrm{~h}$. Western blot analysis was performed to detect $\mathrm{NF}-\kappa \mathrm{B}$ protein expression in the nuclear fraction and p53 protein expression in total cells. (D) Quantitative analysis of the protein expression. Values represent the means \pm SD of three independent experiments. ${ }^{*} \mathrm{P}<0.05$ vs. control; ${ }^{*} \mathrm{p}<0.05$ vs. MEG3. MEG3, maternally expressed gene 3 ; Lv-MEG3, lentiviral vector containing MEG3.

nofluorescence and immunohistochemistry assays showed that ectopic expression of MEG3 obviously caused NF- $\kappa$ B translocation from the cytoplasm to the nucleus and increased its expression in nuclei (Fig. 5A and B). Furthermore, western blot analysis demonstrated that the majority of the NF- $\kappa B$ proteins resided in the nucleus and the expression level of PERK was also increased in the MEG3-transfected cells (Fig. 5C), indi- cating that the PERK/eIF2a/NF- $\mathrm{B}$ pathway was activated. Moreover, inhibition of NF- $\kappa \mathrm{B}$ by Bay11-7082 decreased $\mathrm{p} 53$ protein expression (Fig. 5C and D), showing that p53 expression was regulated by $\mathrm{NF}-\kappa \mathrm{B}$ under ER stress. Our previous study identified that MEG3 repressed MDM2 expression and induced p53 activation (10). Recently, Zhu et al demonstrated that MEG3 can also interact directly with the p53 DNA 
binding domain in hepatoma cells (29). In the present study, we observed that overexpression of MEG3 activated the ER stress pathway and increased p53 expression via the $\mathrm{NF}-\kappa \mathrm{B}$ pathway, which indicates that multiple mechanisms participate in p53 activation in MEG3-induced apoptosis.

The role of $N F-\kappa B$ in regulating cell survival or death is complex. NF- $\kappa \mathrm{B}$ mediates tumor promotion, angiogenesis, metastasis, and resistance to chemotherapeutics (30). In a study of gastric carcinoma, we demonstrated that NF- $\mathrm{BB}$ was constitutively active and associated with advanced pathologic stage and tumor size (31). In adenosine-induced apoptosis, we observed that $\mathrm{NF}-\kappa \mathrm{B}$ plays an anti-apoptotic role (32). However, in the present study, unexpectedly, inhibition of $\mathrm{NF}-\kappa \mathrm{B}$ decreased p53 protein expression. We hypothesize that the difference may be due to the types of stress and the features of the cell lines. ER stress can operate in parallel with multiple signaling mechanisms in different situations, and the overall outcome in terms of cell survival or apoptosis depends on the additive effects on downstream effectors. The role of $\mathrm{NF}-\kappa \mathrm{B}$ on $\mathrm{p} 53$ regulation needs further investigation in MEG3-mediated ER stress.

In summary, the present study demonstrated that exogenous MEG3 impeded tumorigenesis both in vitro and in vivo and induced hepatoma cell apoptosis. Moreover, overexpression of MEG3 caused ER stress and resulted in the activation of $\mathrm{NF}-\kappa \mathrm{B}$ and $\mathrm{p} 53$. Furthermore, inhibition of $\mathrm{NF}-\kappa \mathrm{B}$ decreased p53 protein expression. These results showed that the ER stress pathway may be involved in MEG3-induced apoptosis and that $\mathrm{NF}-\kappa \mathrm{B}$ signaling is required for $\mathrm{p} 53$ activation in ER stress.

\section{Acknowledgements}

This study was supported by the Guangdong Natural Science Foundation in China (no. 2014A030313470) and the Collaborative and Creative Center, Molecular Diagnosis and Personalized Medicine, Shantou University, Guangdong, China. This study was also supported by the Department of Education, Guangdong Government under the Top-tier University Development Scheme for Research and Control of Infectious Diseases.

\section{References}

1. Wapinski $\mathrm{O}$ and Chang HY: Long noncoding RNAs and human disease. Trends Cell Biol 21: 354-361, 2011.

2. Wang P, Ren Z and Sun P: Overexpression of the long non-coding RNA MEG3 impairs in vitro glioma cell proliferation. J Cell Biochem 113: 1868-1874, 2012.

3. Li Z, Li C, Liu C, Yu S and Zhang Y: Expression of the long non-coding RNAs MEG3, HOTAIR, and MALAT-1 in non-functioning pituitary adenomas and their relationship to tumor behavior. Pituitary 18: 42-47, 2015

4. Miyoshi N, Wagatsuma H, Wakana S, Shiroishi T, Nomura M, Aisaka K, Kohda T, Surani MA, Kaneko-Ishino T and Ishino F: Identification of an imprinted gene, Meg3/Gt12 and its human homologue MEG3, first mapped on mouse distal chromosome 12 and human chromosome 14q. Genes Cells 5: 211-220, 2000.

5. Zhou Y, Zhang X and Klibanski A: MEG3 noncoding RNA: A tumor suppressor. J Mol Endocrinol 48: R45-R53, 2012.

6. Bando T, Kato Y, Ihara Y, Yamagishi F, Tsukada K and Isobe M: Loss of heterozygosity of $14 \mathrm{q} 32$ in colorectal carcinoma. Cancer Genet Cytogenet 111: 161-165, 1999.

7. Lu KH, Li W, Liu XH, Sun M, Zhang ML, Wu WQ, Xie WP and Hou YY: Long non-coding RNA MEG3 inhibits NSCLC cells proliferation and induces apoptosis by affecting p53 expression. BMC Cancer 13: 461, 2013 .
8. Braconi C, Kogure T, Valeri N, Huang N, Nuovo G, Costinean S, Negrini M, Miotto E, Croce CM and Patel T: microRNA-29 can regulate expression of the long non-coding RNA gene MEG3 in hepatocellular cancer. Oncogene 30: 4750-4756, 2011.

9. Zhang X, Gejman R, Mahta A, Zhong Y, Rice KA, Zhou Y, Cheunsuchon P, Louis DN and Klibanski A: Maternally expressed gene 3, an imprinted noncoding RNA gene, is associated with meningioma pathogenesis and progression. Cancer Res 70: 2350-2358, 2010.

10. Liu LX, Deng W, Zhou XT, Chen RP, Xiang MQ, Guo YT, Pu ZJ, Li R, Wang GF and Wu LF: The mechanism of adenosine-mediated activation of lncRNA MEG3 and its antitumor effects in human hepatoma cells. Int J Oncol 48: 421-429, 2016.

11. Schröder M: Endoplasmic reticulum stress responses. Cell Mol Life Sci 65: 862-894, 2008.

12. Wu LF, Ye YQ, Huang GY, Li HB, Li GP, Pu ZJ, Wei BL and Feng JL: Involvement of endoplasmic reticulum stress in adenosine-induced human hepatoma HepG2 cell apoptosis. Oncol Rep 26: 73-79, 2011.

13. Giampietri C, Petrungaro S, Conti S, Facchiano A, Filippini A and Ziparo E: Cancer microenvironment and endoplasmic reticulum stress response. Mediators Inflamm 2015: 417281, 2015.

14. Wu LF, Guo YT, Zhang QH, Xiang MQ, Deng W, Ye YQ, $\mathrm{Pu} \mathrm{ZJ}$, Feng JL and Huang GY: Enhanced antitumor effects of adenoviral-mediated siRNA against GRP78 gene on adenosine-induced apoptosis in human hepatoma HepG2 cells. Int J Mol Sci 15: 525-544, 2014.

15. Zinszner H, Kuroda M, Wang X, Batchvarova N, Lightfoot RT, Remotti H, Stevens JL and Ron D: CHOP is implicated in programmed cell death in response to impaired function of the endoplasmic reticulum. Genes Dev 12: 982-995, 1998.

16. Nakagawa T, Zhu H, Morishima N, Li E, Xu J, Yankner BA and Yuan J: Caspase-12 mediates endoplasmic-reticulum-specific apoptosis and cytotoxicity by amyloid-beta. Nature 403: 98-103, 2000.

17. Urano F, Wang X, Bertolotti A, Zhang Y, Chung P, Harding HP and Ron D: Coupling of stress in the ER to activation of JNK protein kinases by transmembrane protein kinase IRE1. Science 287: 664-666, 2000.

18. Zhou Y, Zhong Y, Wang Y, Zhang X, Batista DL, Gejman R, Ansell PJ, Zhao J, Weng C and Klibanski A: Activation of p53 by MEG3 non-coding RNA. J Biol Chem 282: 24731-24742, 2007.

19. Yang PM, Lin YT, Shun CT, Lin SH, Wei TT, Chuang SH, Wu MS and Chen CC: Zebularine inhibits tumorigenesis and stemness of colorectal cancer via p53-dependent endoplasmic reticulum stress. Sci Rep 3: 3219, 2013.

20. Kilkenny C, Browne W, Cuthill IC, Emerson M and Altman DG; NC3Rs Reporting Guidelines Working Group: Animal research: Reporting in vivo experiments: The ARRIVE guidelines. Br J Pharmacol 160: 1577-1579, 2010.

21. Lee AS: The ER chaperone and signaling regulator GRP78/BiP as a monitor of endoplasmic reticulum stress. Methods 35: 373-381, 2005 .

22. Bromati CR, Lellis-Santos C, Yamanaka TS, Nogueira TC, Leonelli M, Caperuto LC, Gorjão R, Leite AR, Anhê GF and Bordin S: UPR induces transient burst of apoptosis in islets of early lactating rats through reduced AKT phosphorylation via ATF4/CHOP stimulation of TRB3 expression. Am J Physiol Regul Integr Comp Physiol 300: R92-R100, 2011.

23. Wu H and Lozano G: NF-kappa B activation of p53. A potential mechanism for suppressing cell growth in response to stress. J Biol Chem 269: 20067-20074, 1994.

24. Furlong EE, Rein T and Martin F: YY1 and NF1 both activate the human 553 promoter by alternatively binding to a composite element, and YY1 and E1A cooperate to amplify p53 promoter activity. Mol Cell Biol 16: 5933-5945, 1996.

25. Kitamura M: Control of NF- $\kappa B$ and inflammation by the unfolded protein response. Int Rev Immunol 30: 4-15, 2011.

26. Kaneko M, Niinuma Y and Nomura Y: Activation signal of nuclear factor-kappa B in response to endoplasmic reticulum stress is transduced via IRE1 and tumor necrosis factor receptor-associated factor 2. Biol Pharm Bull 26: 931-935, 2003.

27. Jiang HY, Wek SA, McGrath BC, Scheuner D, Kaufman RJ, Cavener DR and Wek RC: Phosphorylation of the alpha subunit of eukaryotic initiation factor 2 is required for activation of NF-kappaB in response to diverse cellular stresses. Mol Cell Biol 23: 5651-5663, 2003. 
28. Yamazaki H, Hiramatsu N, Hayakawa K, Tagawa Y, Okamura M, Ogata R, Huang T, Nakajima S, Yao J, Paton AW, et al: Activation of the Akt-NF-kappaB pathway by subtilase cytotoxin through the ATF6 branch of the unfolded protein response. J Immunol 183: 1480-1487, 2009

29. Zhu J, Liu S, Ye F, Shen Y, Tie Y, Zhu J, Wei L, Jin Y, Fu H, $\mathrm{Wu} \mathrm{Y}$, et al: Long noncoding RNA MEG3 interacts with p53 protein and regulates partial p53 target genes in hepatoma cells. PLoS One 10: e0139790, 2015.

30. Pikarsky E, Porat RM, Stein I, Abramovitch R, Amit S Kasem S, Gutkovich-Pyest E, Urieli-Shoval S, Galun E and Ben-Neriah Y: NF-kappaB functions as a tumour promoter in inflammation-associated cancer. Nature 431: 461-466, 2004.
31. Wu L, Pu Z, Feng J, Li G, Zheng Z and Shen W: The ubiquitin-proteasome pathway and enhanced activity of NF-kappaB in gastric carcinoma. J Surg Oncol 97: 439-444, 2008.

32. Wu LF, Li GP, Su JD, Pu ZJ, Feng JL, Ye YQ and Wei BL: Involvement of NF-kappaB activation in the apoptosis induced by extracellular adenosine in human hepatocellular carcinoma HepG2 cells. Biochem Cell Biol 88: 705-714, 2010. 